\title{
étude théorique et expérimentale du fluage unidimensionnel des sols argileux
}

\author{
par \\ B. Félix \\ Ingénieur au Département Sols et Fondations du Laboratoire Central des Ponts et Chaussées
}

\begin{abstract}
Le fluage des sols fins est dû aux propriétés visqueuses de l'assemblage des particules minérales entourées d'eau adsorbée qui forme le squelette intergranulaire. II apparaît avec évidence au cours de la consolidation secondaire : les contraintes effectives étant constantes, la déformation se prolonge dans le temps.

La première fonction d'un modèle mathématique du fluage est d'établir une relation entre cette déformation, la contrainte constante et le temps. Mais la viscosité du squelette intervient quel que soit le régime de chargement. Elle influe, par exemple sur le développement de la consolidation hydrodynamique,
\end{abstract}

au cours de laquelle les contraintes effectives croissent lentement, ou sur la déformation d'un sol de fondation progressivement chargé par un ouvrage en construction. Dans ce cas, le modèle de fluage doit lier la déformation au temps et à une contrainte variable, elle-même fonction du temps. II peut alors constituer l'équation d'état du squelette intergranulaire dans une théorie de la consolidation.

Pour autant le modèle doit être simple et comporter un nombre réduit de paramètres faciles à déterminer à partir des données de l'expérience. C'est la constitution d'un tel modèle qui va être abordée.

\section{Première partie : Comparaison des modèles du fluage unidimensionnel}

\section{Généralités}

L'usage réservé aux modèles du fluage unidimensionnel n'est pas sans influence sur leur forme mathématique.

S'il s'agit de décrire correctement le fluage des sols tel qu'il est observé au laboratoire ou in situ sous des contraintes imposées, il est intéressant d'obtenir à l'aide du modèle la reproduction la plus précise possible des résultats mesurés. La forme mathématique est choisie par compromis entre le degré de cette précision et la complexité du modèle (le nombre de ses paramètres). Le fluage des matériaux dépourvus de phase liquide tels que l'acier, le béton, les polymères, etc., est décrit en suivant cette démarche, qui peut être qualifiée de phénoménologique.

Si le modèle du fluage unidimensionnel est intégré dans une théorie de la consolidation, il représente alors l'équation d'état du squelette intergranulaire doué de viscosité. Certaines formes mathématiques permettent plus facilement que d'autres, une résolution du problème. Il est tentant de la choisir a priori, en la justifiant parfois par des considérations sur le mécanisme de déformation des liaisons entre grains.
Une méthode de détermination des paramètres du modèle, à partir des résultats d'essais œdométriques, est quelquefois proposée; mais rares sont les vérifications expérimentales de sa validité. Le comportement du squelette est supposé analogue à celui d'un modèle idéal, la démarche est analogique.

La frontière entre les deux démarches que nous venons de distinguer s'est quasiment évanouie avec l'apparition de l'ordinateur et des méthodes de résolution numérique du problème de la consolidation, qui permettent, en effet, contrairement aux méthodes analytiques traditionnelles, une grande liberté dans le choix des lois de comportement.

\section{Les corps idéaux}

\subsection{Les corps idéaux linéaires}

Les premières simulations du comportement visqueux du squelette au cours de la consolidation, ont été effectuées à l'aide de corps idéaux classiques, formés de l'assemblage en série et en parallèle de ressorts et d'amortisseurs à caractéristiques constantes. 
Taylor (1940) dans sa théorie A de la consolidation, a utilisé le corps idéal de Hohenemser-Prager (fig. 1a) dont l'équation différentielle de comportement s'écrit :

$$
\left(E+E_{1}\right) \sigma^{\prime}(t)+\eta \dot{\sigma}^{\prime}(t)=E E_{1} \varepsilon(t)+\eta E \dot{\varepsilon}(t) .
$$

Si le régime de chargement $\sigma^{\prime}(\mathrm{t})$ est imposé et respecte les conditions $\sigma^{\prime}=\varepsilon=0$ pour $t=0$, l'équation (1) devient l'équation de fluage (2) par transformation de Laplace :

$$
\varepsilon(t)=\frac{\sigma^{\prime}(t)}{E}+\frac{1}{\eta} \int_{0}^{t} \sigma^{\prime}(\tau) e^{-\frac{E_{1}}{\eta}(t-\tau)} d \tau .
$$

Par contre, Taylor (1942) a retenu dans sa théorie B de la consolidation le corps idéal plus simple de Kelvin-Voigt (fig. 1 b) dont l'équation différentielle de comportement s'écrit :

$$
\sigma^{\prime}(t)=E \varepsilon(t)+\eta \dot{\varepsilon}(t),
$$

et l'équation de fluage,

$$
\varepsilon(t)=\frac{1}{\eta} \int_{0}^{t} \sigma^{\prime}(\tau) e^{-\frac{\epsilon}{\eta}(t-x)} \mathrm{d} \tau .
$$

La déformation du premier modèle sous un palier de charge est en partie instantanée et en partie différée: alors que celle du deuxième modèle est entièrement différée. Les deux hypothèses se rencontrent sur les modèles examinés par la suite. Laquelle des deux correspond à la réalité physique? Cette question n'a jamais pu être tranchée par l'expérience.

La théorie A de Taylor (1940) a été complétée par Tan (1957) et surtout Gibson et Lo (1961) qui lui ont trouvé une solution analytique.

La similitude entre le squelette intergranulaire d'un sol argileux et les corps idéaux linéaires à deux ou trois éléments est très approximative. Pour y remédier, Schiffman (1969) a multiplié le nombre des éléments à caractéristique constante, en plaçant en série avec un corps de Hohenemser-Prager (fig. 1 a) un nombre $n$ fini de corps de Kelvin-Voigt (fig. $1 \mathrm{~b}$ ). II a écrit l'équation de fluage de son modèle de la manière suivante:

$$
\varepsilon(t)=\frac{\sigma^{\prime}(t)}{E}+\int_{0}^{t}\left[\sum_{i=1}^{n} \frac{1}{\eta_{i}} e^{-\frac{E_{i}}{\eta_{i}}(t-\tau)}\right] \sigma^{\prime}(\tau) d \tau .
$$

\subsection{Les corps idéaux non linéaires}

Pour mieux reproduire le comportement réel du squelette à l'aide de corps idéaux, d'autres auteurs ont proposé de les doter de caractéristiques variables.

D'après Barden (1965), la viscosité de l'amortisseur placé dans le corps de Kelvin-Voigt varie avec la vitesse de déformation; l'équation différentielle de comportement s'écrit :

$$
\sigma^{\prime}(t)=E \varepsilon(t)+b[\dot{\varepsilon}(t)]^{\frac{1}{n}} .
$$

Vu Cao Minh (1977) a proposé une méthode de détermination des paramètres $b$ et $n$ de ce modèle. Sous une charge constante $\sigma_{0}^{\prime}$, le corps présente une déformation finale égale à $\varepsilon_{\mathrm{f}}=\frac{\sigma_{\mathrm{o}}^{\prime}}{\mathrm{E}}$. La différence entre

celle-ci et la déformation au temps t est liée à la vitesse de déformation par une loi puissance. La droite qui la représente dans un diagramme bilogarithmique a une pente $n$.

Wu et al (1966) ont décrit la vitesse de déformation de l'amortisseur du corps idéal de Poynting-Thompson (fig. 1c), conformément à la théorie des vitesses absolues de réaction (rate process theory), élaborée pour les sols par Murayama et Shibata (1964). Elle est liée à la contrainte appliquée à l'amortisseur par la

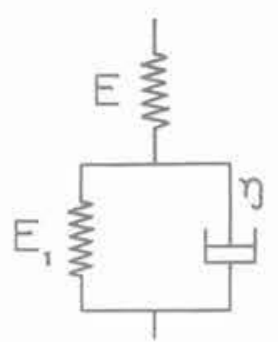

a

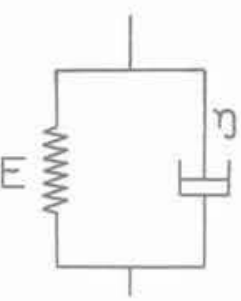

b

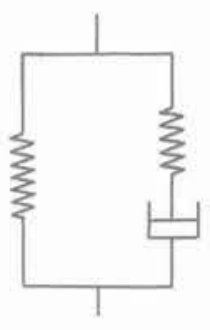

C
Fig. 1 Schémas des corps idéaux

relation (7) :

$$
\varepsilon_{\mathrm{a}}=\lambda \operatorname{sh}\left(\mu \sigma_{\mathrm{a}}^{\prime}\right) \text {. }
$$

Barden (1969) a introduit les mêmes caractéristiques pour l'amortisseur du corps idéal de Kelvin-Voigt.

Tous ces corps idéaux non linéaires ont une compressibilité constante. Par contre Poskitt et Birdsall (1970) ont modifié le corps de HohenemserPrager, doté d'un amortisseur obéissant à la loi (7), de façon à faire varier, avec le degré de déformation de chaque élément, la compressibilité des ressorts ainsi que les paramètres de la loi (7). Uitérieurement Poskitt (1971) a transformé de la même façon le corps idéal de Kelvin-Voigt.

Le nombre de paramètres de ces modèles est bien entendu plus grand que celui de leur version linéaire. Le problème de leur détermination expérimentale n'a pas été résolu, sinon pour le modèle de Barden (1965) par Vu Cao Minh.

\section{Lois de la forme $\varepsilon=f\left(\sigma^{\prime}, t\right)$}

3.1 Les lois logarithmiques de cette forme se prêtent bien a une approche phénoménologique du fluage unidimensionnel, car il a été remarqué après Pokrovskij (1933) et Buisman (1936) que la déformation "secondaire" d'un sol sous une charge constante, variait linéairement avec le logarithme du temps.

Selon Buisman, le tassement d'un horizon d'épaisseur ho, soumis à une pression uniforme $P$, est égal à :

$$
s(t)=h o\left(\alpha_{p}+\alpha_{s} \lg t\right) P \text {. }
$$

Koppejan (1948), compte tenu de la loi logarithmique établie entre contrainte et déformation par K. Terzaghi, a proposé :

$$
\varepsilon(t)=\left[\frac{1}{C_{c p}}+\frac{1}{C_{c s}} \lg t\right] \lg \frac{\sigma_{0}^{\prime}+\Delta \sigma^{\prime}}{\sigma_{0}^{\prime}} .
$$

Un mode opératoire existe pour la détermination des paramètres $C_{c p}$ et $C_{c s}$. Dans la relation (10) les fonctions du temps et de la contrainte sont multipliées; cette loi est multiplicative.

Dans la pratique courante, une loi additive lui est généralement préférée. La déformation est la somme d'un terme pseudo-instantané fonction de la contrainte, et d'un terme fonction du temps de la forme suivante :

$$
\Delta \mathrm{e}=-\mathrm{C}_{\alpha} \lg \mathrm{t} \text {. }
$$

Le taux de consolidation secondaire $\mathrm{C}_{\alpha}$ caractérise ce dernier terme, tout comme l'indice de compression $\mathrm{C}_{\mathrm{o}}$ caractérise le premier. Mesri et Godlewski (1976) ont, pour quelques argiles caractéristiques, lié ces deux paramètres par une corrélation. Les expériences de 
Leonards et Girault (1961) ont montré l'influence du taux d'accroissement de la charge sur $\mathrm{C}_{\alpha}$; celles de Mieussens (1979) indiquent dans certaines conditions une variation de ce paramètre dans le temps.

Bjerrum (1967) a proposé un modèle de la forme additive précédente. L'indice des vides du matériau est lié à la contrainte par une courbe, appelée " time-line" par Taylor, et qui est caractérisée par la durée d'application de cette contrainte. Aux diverses valeurs du temps correspond un faisceau de courbes parallèles qui sont représentées sur la figure 2 .

Un sol argileux préconsolidé sous une charge $\sigma_{0}^{\prime}$ pendant un temps $t_{\infty}$ subit, sous un incrément de charge $\Delta \sigma^{\prime}$, une déformation que Bjerrum décompose en trois termes. La composante quasi-instantanée est répartie entre les domaines de comportement surconsolidé et normalement consolidé. Le premier est caractérisé par une compressibilité $\mathrm{C}_{\mathrm{s}}$, beaucoup plus petite que celle appelée $C_{c}$, qui caractérise le second. La contrainte $\sigma_{p}^{\prime}$ de quasi-préconsolidation qui marque la frontière, est fonction de la durée $\mathrm{t}_{\infty}$ d'application de la charge $\sigma_{0}^{\prime}$ de préconsolidation :

$$
e_{t}-e_{0}=C_{s} \lg \frac{\sigma_{p}^{\prime}}{\sigma_{o}^{\prime}}+C_{c} \lg \frac{\sigma_{0}^{\prime}+\Delta \sigma^{\prime}}{\sigma_{p}^{\prime}}+C_{\alpha} \lg \frac{t_{\infty}+t}{t_{\infty}} \text {. }
$$

Ce modèle a été utilisé par Magnan et al (1979) pour traiter numériquement, par le programme CONMULT. la consolidation unidimensionnelle d'un multicouche.

Mesri et Rokhsar (1974) ont supposé que la déformation différée est proportionnelle au logarithme du temps, mais que le taux de consolidation secondaire variait linéairement avec la déformation. II est nul au moment du chargement et égal à $C_{\alpha}$ à la fin de la consolidation primaire.

\subsection{Lois non logarithmiques}

Hansen (1969) a représenté les « time-lines * de Taylor par des droites dans un diagramme bilogarithmique; dans ces conditions, l'équation (11) de Bjerrum devient :

$$
\frac{\mathrm{e}}{\mathrm{e}_{\mathrm{o}}}=\left[\frac{\sigma_{\mathrm{p}}^{\prime}}{\sigma_{0}^{\prime}}\right]^{-\mathrm{a}}\left[\frac{\sigma_{0}^{\prime}+\Delta \sigma^{\prime}}{\sigma_{\mathrm{p}}^{\prime}}\right]^{-\mathrm{b}}\left[\frac{\mathrm{t}_{\infty \mathrm{s}}+\mathrm{t}}{\mathrm{t}_{\infty}}\right]^{-\mathrm{o}} .
$$

Garlanger (1972) a décrit le comportement du squelette intergranulaire au cours de la consolidation à l'aide de ce modèle, qui est multiplicatif.

Meschyan (1967) a cité et utilisé un grand nombre de lois de fluage multiplicatives de la forme:

$$
\varepsilon=\mathrm{C}(\mathrm{t}) \quad \mathrm{f}\left(\sigma^{\prime}\right) .
$$

La plupart de ces fonctions du temps et de la contrainte, sont issues de l'étude du fluage d'autres matériaux, tels que le béton, I'acier, etc.

En général, comme le fait observer Meschyan, les lois de la forme $\varepsilon=f\left(\sigma^{\prime}, t\right)$ rendent imparfaitement compte de la déformation provoquée par une charge qui varie dans le temps. Car, à un instant donné où la contrainte est égale à $\sigma^{\prime}(\mathrm{t})$, la déformation est supposée égale à celle que provoquerait la même charge maintenue constante depuis le début du chargement.

\section{Lois de la forme $\dot{\varepsilon}=f\left(\sigma^{\prime}, \varepsilon\right)$}

Le temps ne figure pas dans l'expression mathématique précédente. Et la vitesse de déformation est la seule dérivée par rapport au temps qui est représentée.

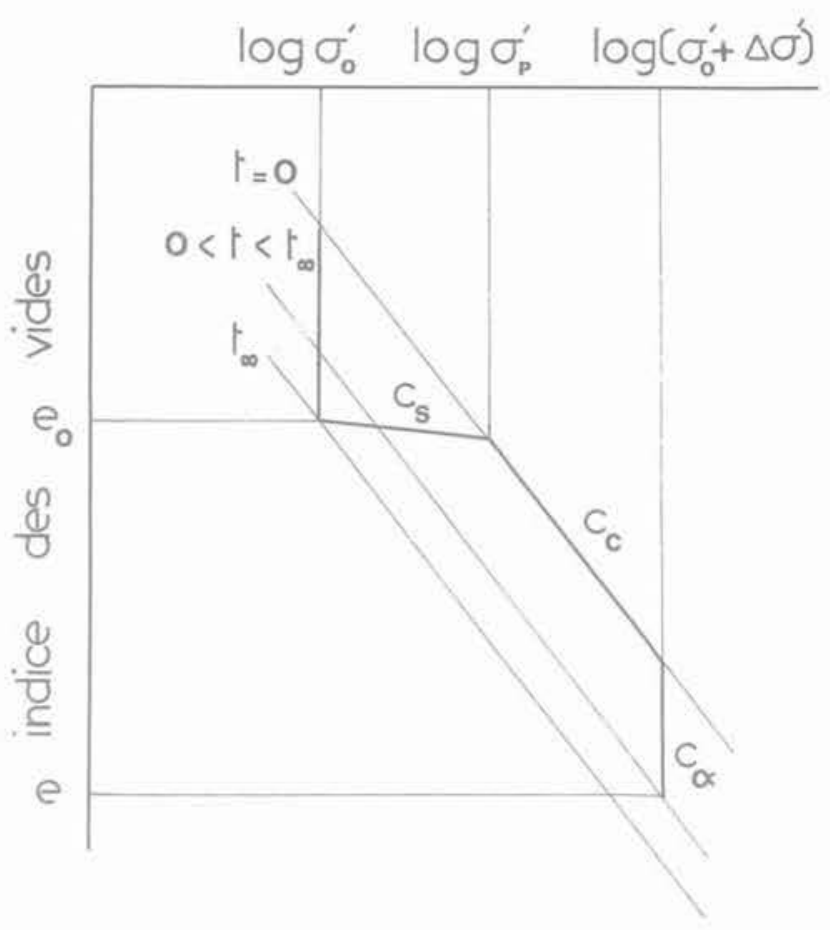

Fig. 2 Décomposition du tassement d'après L. Bjerrum

Battelino (1973) a fait observer que tous les corps idéaux, linéaires ou non, dont la déformation instantanée sous un incrément de charge est nulle, admettent une loi de comportement de la forme (14). Les équations (3) et (6) des modèles de Kelvin-Voigt et de Barden en sont un exemple.

Les lois de la forme (14) sont associées à la notion d'isotaches proposées par Šuklje (1957). Les courbes isotaches forment un faisceau qui représente le comportement du squelette intergranulaire. Chacune d'elles, d'équation :

$$
F\left(\sigma^{\prime}, e\right)=\dot{e}_{\text {consts }}
$$

représente l'ensemble des états de contraintedéformation, caractérisés par $\sigma^{\prime}$ et e, qui se traduisent par la même vitesse de variation de l'indice des vides $\dot{\mathrm{e}}_{\text {canst. }}$.

Ultérieurement, Šuklje a proposé plusieurs équations de courbes isotaches qui ont servi dans la résolution numérique de divers problèmes de consolidation :

$\frac{\dot{\mathrm{e}}}{\dot{\dot{e}_{0}}}=\exp \frac{\mathrm{A}+\mathrm{B} \ln \frac{\sigma^{\prime}}{\sigma_{0}^{\prime}}-\mathrm{e}}{\mathrm{C}+\mathrm{D} \ln \frac{\sigma^{\prime}}{\sigma_{0}^{\prime}}}$ Suklje Kozak (1974)

$\dot{\mathrm{e}}=c A e^{\frac{c+1}{c}} \sigma^{\prime}$ Š Šklje Kovačič (1974)

où $\mathrm{A}, \mathrm{B}, \mathrm{C}, \mathrm{D}$ et $\mathrm{c}, \mathrm{d}$ sont des constantes caractéristiques du matériau.

D'autres équations du type (15), ont été utilisées dans une théorie de la consolidation par des auteurs, qui n'ont cependant pas fait référence à la méthode des isotaches :

$$
\dot{e}=-\frac{a\left(\sigma^{\prime}-\sigma_{0}^{\prime}\right)^{n}}{k}\left[e-e_{0} \frac{\left(\sigma^{\prime}-\sigma_{0}^{\prime}\right)^{m}}{E}\right] .
$$

II s'agit pour l'équation (18) de Poorooshasb et Sivapatham (1969) et pour la suivante (19) de Hawley et Borin (1973) :

$$
\dot{e}=B \frac{\Gamma_{u}+\lambda_{u} \lg \sigma^{\prime}-e}{\Gamma_{L}+\lambda_{L} \lg \sigma^{\prime}-e}
$$

$a, k, E, m$ et $\Gamma_{u}, \lambda_{u}, \Gamma_{L}, \lambda_{L}$ sont ici aussi des constantes. 
Les corps dont la loi de comportement est de la forme (14) ont la propriété suivante : soumis à des contraintes identiques et présentant la même déformation, ils possèdent la même vitesse de fluage. Quelle que soit I'histoire antérieure du chargement, le développement de leur déformation sera identique.

La figure 3 schématise, en fonction du temps, l'évolution des déformations provoquées par la même charge, appliquée en une seule fois ou en deux. La portion de courbe relative au deuxième palier se déduit par translation suivant $\mathrm{O}^{\prime} \mathrm{A}$ de la portion de courbe relative à la charge constante, pourvu que la déformation initiale soit la même.

Meschyan (1967) a vérifié cette propriété de la déformation de fluage des sols argileux les plus divers sans formuler de loi mathématique du type (14).

\section{Théorie du fluage héréditaire}

Le principe de superposition, employé en théorie de l'élasticité, a été généralisé aux corps visco-élastiques par Boltzmann en 1876.

Une charge $\sigma^{\prime}(\tau)$, appliquée au temps $\tau$ durant un court intervalle $\Delta \tau$, provoque après un temps $t-\tau$, une déformation différée égale à :

$$
\varepsilon(\mathrm{t})=\mathrm{K}(\mathrm{t}-\tau) \sigma^{\prime}(\tau) \Delta \tau .
$$

$\mathrm{K}(\mathrm{t})$ est la fonction d'influence; elle tend vers $\mathrm{O}$ lorsque le temps devient infini car la déformation est réversible.

La déformation provoquée par l'application successive de charges momentanées est la somme des déformations provoquées par chacune d'elles, à laquelle s'ajoute la déformation élastique instantanée due à la charge $\sigma^{\prime}(\mathrm{t})$ appliquée au moment de l'observation :

$$
\varepsilon(t)=\frac{\sigma^{\prime}(t)}{E}+\sum_{i=1}^{n} K\left(t-\tau_{i}\right) \sigma^{\prime}\left(\tau_{i}\right) \Delta \tau_{e} .
$$

Lorsque $\sigma^{\prime}(t)$ varie continûment, on obtient :

$$
\varepsilon(t)=\frac{\sigma^{\prime}(t)}{E}+\int_{0}^{t} K(t-\tau) \sigma^{\prime}(\tau) d \tau .
$$

Cette équation (22) est l'équation d'état de la théorie du fluage héréditaire linéaire.

Si la charge est constante et égale à l'unité $\sigma_{1}^{\prime}=1$, on obtient :

$$
\varepsilon(t)=C(t)=\frac{1}{E}+\int_{0}^{t} K(t-\tau) d \tau=\frac{1}{E}+\int_{0}^{t} K(\tau) d \tau .
$$

La fonction $\mathrm{C}(\mathrm{t})$ est appelée mesure de fluage; sa dérivée $K(t)$ est donc la vitesse de fluage sous une charge unité.

Les corps idéaux linéaires constituent des cas particuliers de la théorie du fluage héréditaire linéaire. Leurs équations de fluage (2) (4) et (5) sont en effet de la forme (22), avec des fonctions d'influence qui sont des termes exponentiels du temps ou des sommes de termes exponentiels (5).

Florin (1953) puis Ter Martirosyan (1965) et Zaretskij (1967) ont utilisé, dans diverses théories de la consolidation uni ou tridimensionnelles la forme (22) de l'équation d'état du squelette intergranulaire, avec des fonctions d'influence de type exponentiel. En conséquence, les modèles qu'ils ont utilisés sont identiques aux corps idéaux linéaires.

La théorie du fluage héréditaire peut être généralisée aux matériaux dont les propriétés, caractérisées par la fonction $K(t, \tau)$ et le module élastique $E(t)$ varient avec l'âge.

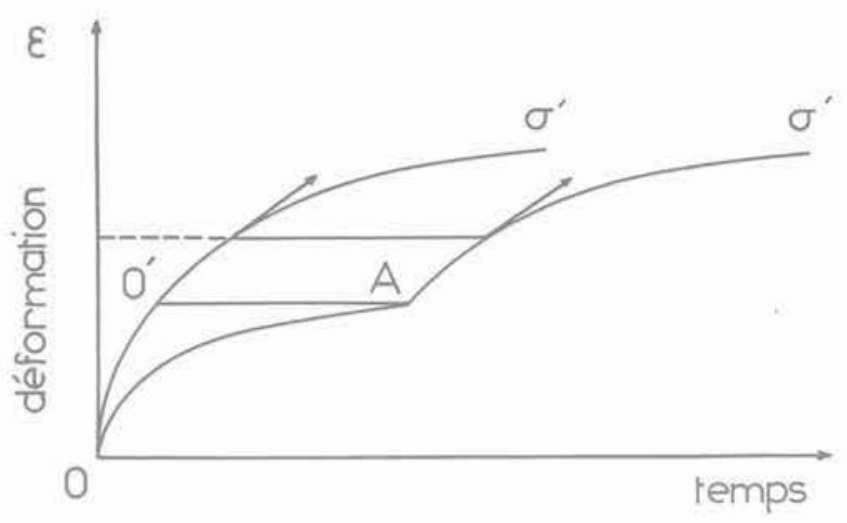

Fig. 3 Translation des courbes d'équation :

$\dot{\varepsilon}=f\left(\sigma^{\prime}, \varepsilon\right)$

Maslov (1940) et Arutyunyan (1951) proposent dans la théorie du corps élastofluant, l'équation suivante de comportement :

$$
\varepsilon(t)=\frac{\sigma^{\prime}(t)}{E(t)}+\int_{0}^{t} K(t, \tau) \sigma^{\prime}(\tau) d \tau .
$$

Elle peut être également généralisée aux matériaux non linéaires; d'après Robotnov (1948), elle s'écrit alors sous la forme :

$$
\varepsilon(t)=\frac{f\left[\sigma^{\prime}(t)\right]}{E}+\int_{0}^{t} K(t-\tau) f\left[\sigma^{\prime}(\tau)\right] d \tau .
$$

Pour la charge constante unité $\sigma_{1}^{\prime}=1 ; f\left(\sigma_{1}^{\prime}\right)=1$ et la déformation est égale à la mesure de fluage $\mathrm{C}(\mathrm{t})$.

Pour une charge constante quelconque, elle est égale à :

$$
\varepsilon(t)=C(t) f\left(\sigma^{\prime}\right),
$$

c'est-à-dire à une expression du fluage de forme multiplicative identique à (13).

\section{Conclusion}

La théorie du fluage héréditaire possède une certaine généralité puisqu'elle inclut en tant que cas particuliers les corps idéaux linéaires et, pour les chargements constants, les lois de fluage de forme multiplicative. La mesure de fluage et la fonction de contrainte qui interviennent dans l'équation non linéaire ont une signification physique évidente, et la détermination de leurs paramètres est d'une simplicité que seule permet une approche phénoménologique. Nous savons qu'il n'en va pas de même lorsque l'équation de fluage est bâtie sur l'analogie avec un corps idéal. Cependant, malgré son intérêt théorique et pratique, la théorie du fluage héréditaire a été peu utilisée en mécanique des sols. Dans la deuxième partie un modèle issu de cette théorie va être développé. 


\section{Deuxième partie : Fluage héréditaire des sols à viscosité et compressibilité variables}

\section{Introduction}

Choisir un modèle issu de la théorie du fluage héréditaire non linéaire, c'est choisir la forme mathématique de la mesure de fluage et de la fonction de contrainte. Les paramètres de ces fonctions peuvent être déterminés par ajustement avec les déformations observées au cours de la consolidation secondaire d'une série d'échantillons soumis à des charges constantes. Le modèle est avant tout un modèle phénoménologique de la consolidation secondaire; son domaine d'application est en fait "extrapolé " à la phase hydrodynamique de la consolidation.

II est évident que la validité de cette extrapolation, de même que celle des hypothèses de la théorie du fluage héréditaire, demande une vérification expérimentale.

\section{Généralités sur le modèle}

\subsection{Forme mathématique des fonctions $\mathrm{C}(\mathrm{t})$ et $f\left(\sigma^{\prime}\right)$}

- La mesure de fluage $\mathrm{C}(\mathrm{t})$ est la déformation sous une contrainte constante choisie comme unité $\sigma_{1}^{\prime}$.

- La fonction de contrainte $f\left(\sigma^{\prime}\right)$ est le rapport d'affinité indépendant du temps entre la déformation produite par une charge constante quelconque, et celle produite au même moment par la contrainte unité :

$$
f\left(\sigma^{\prime}\right)=\frac{\varepsilon\left(t, \sigma^{\prime}\right)}{C(t)} \text {. }
$$

La mesure de fluage, pour les valeurs infinies du temps, atteint une limite finie car le volume des vides, qui diminue au cours de la déformation, est limité :

$$
\mathrm{C}(\mathrm{t}) \longrightarrow \varepsilon_{\mathrm{t}} \text { quand } \mathrm{t} \longrightarrow \infty .
$$

La vitesse de déformation au moment de l'application du palier de charge unité est infinie et sa variation est continue. Par conséquent, la vitesse de fluage satisfait à la condition :

$$
\frac{\mathrm{d} C(\mathrm{t})}{\mathrm{dt}} \longrightarrow \infty \text { quand } \mathrm{t} \longrightarrow 0 \text {. }
$$

La déformation proprement instantanée du squelette intergranulaire est difficile à mesurer. Elle est supposée négligeable par rapport à la composante différée, quelle que soit la charge appliquée ( $E$ est supposé infini) :

$$
\mathrm{C}(0)=\frac{\sigma_{1}}{\mathrm{E}}=0 .
$$

La mesure, en sollicitation dynamique, des modules de cisaillement instantané de diverses pâtes d'argile très molles, effectuée par différents auteurs, conforte cette hypothèse puisque les valeurs trouvées sont 10 à 50 fois plus grandes que celles des modules statiques correspondants.

La forme mathématique de la mesure de fluage qui satisfait aux trois hypothèses précédentes, est reprise des travaux de Kohlrausch (1863) sur la laine de verre :

$$
C(t)=\varepsilon_{f}\left[1-\exp \left(-\alpha t^{\beta}\right)\right] \text { avec } \beta<1 .
$$

Une fois choisie une valeur conventionnelle du temps $t_{0}$, la fonction de contrainte représente d'après la relation (26), au coefficient $\mathrm{C}\left(\mathrm{t}_{0}\right)$ près, une relation contrainte-déformation classique; elle est linéaire par morceaux

$$
\begin{array}{lll} 
& \text { pour } \sigma^{\prime} \leqslant \sigma_{p}^{\prime} & f\left(\sigma^{\prime}\right)=a_{1} \lg \sigma^{\prime}-b_{1} \\
\text { et } & \text { pour } \sigma^{\prime}>\sigma_{p}^{\prime} & f\left(\sigma^{\prime}\right)=a_{2} \lg \sigma^{\prime}-b_{2}
\end{array}
$$

$\sigma_{\mathrm{p}}^{\prime}$ est la contrainte de préconsolidation.

Les quatre coefficients des relations (31) satisfont aux conditions suivantes :

$\sigma_{o}^{\prime}$ étant la contrainte avant chargement :

$$
a_{1} \text { Ig } \sigma_{0}^{\prime}-b_{1}=0
$$

$\sigma_{1}^{\prime}$ étant la contrainte unité $\left(\sigma_{1}^{\prime}>\sigma_{\mathrm{p}}^{\prime}\right.$ par ex.)

$$
a_{2} \lg \sigma_{1}^{\prime}-b_{2}=1
$$

et pour $\sigma_{p}^{\prime}$ la condition de continuité s'écrit

$$
a_{1} \lg \sigma_{p}^{\prime}-b_{1}=a_{2} \lg \sigma_{p}^{\prime}-b_{2} \text {. }
$$

\subsection{Détermination des paramètres}

La déformation finale $\varepsilon_{t}$, valeur asymptotique de la fonction $\mathrm{C}(\mathrm{t})$, est supposée connue.

Les paramètres $\alpha$ et $\beta$ de la fonction $(30)$ sont déterminés par une méthode de moindre carré des écarts entre $n$ déformations de consolidation secondaire mesurées sous la contrainte unité $\sigma_{1}^{\prime}$ et les déformations calculées correspondantes:

ou

$$
\begin{aligned}
& \sum_{i=1}^{n}\left\{\varepsilon_{i}-\varepsilon_{t}\left[1-\exp \left(-\alpha t_{i}^{\beta}\right)\right]\right\}_{\text {minimal }}^{2} \\
& \sum_{i=1}^{n}\left\{\frac{\varepsilon_{i}-\varepsilon_{i}}{\varepsilon_{f}}-\exp \left(-\alpha t_{i}^{\beta}\right)\right\}_{\text {minimal }}^{2}
\end{aligned}
$$

Pour rendre le calcul plus facile, le critère précédent est abandonné au profit d'un autre, formulé en transformant deux fois les termes dont on calcule la différence, par une fonction monotone croissante; la fonction logarithme :

$$
\begin{aligned}
& \sum_{i=1}^{n}\left\{-\ln \left(\ln \frac{\varepsilon_{f}}{\varepsilon_{f}-\varepsilon_{i}}\right)+\ln \left(\alpha t_{i}^{\beta}\right)\right\}_{\text {minimal }}^{2}= \\
& \sum_{i=1}^{n}\left\{\ln \left(\ln \frac{\varepsilon_{f}}{\varepsilon_{f}-\varepsilon_{i}}\right)-\beta \ln t_{i}-\ln \alpha\right\}_{\text {minimal }}^{2}
\end{aligned}
$$

Cette transformation du critère est équivalente à celle de la variable $t_{i}$ en In $t_{i 1}$ et de la déformation $\varepsilon_{i}$ en $\ln \left(\ln \frac{\varepsilon_{f}}{\varepsilon_{f}-\varepsilon_{i}}\right)$. La pente de la droite des moindres carrés est égale à $\beta$, et son ordonnée à l'origine à ln $\alpha$.

Pour 4 charges constantes considérées comme unité, les mesures de fluage de la forme $(30)$ sont tracées en coordonnées arithmétiques sur la figure 4 , et en coordonnées semi-logarithmiques sur la figure 5 où elles présentent toutes un point d'inflexion. Au voisinage de la valeur du temps correspondante, la fonction de Kohlrausch est quasiment équivalente à la loi logarithmique de Buisman. Pour que cette valeur du temps soit située dans l'intervalle pendant lequel les mesures sont généralement effectuées, la période de grande divergence a été placée au-delà. La déformation finale $\varepsilon_{\mathrm{f}}$ a été calculée comme égale à celle fournie au bout de 100 ans, par la loi logarithmique déterminée par la première déformation de consolidation secondaire $\varepsilon_{1}$ mesurée au temps $t_{1}$, et par la dernière $\varepsilon_{n}$ mesurée au temps $t_{n}$, soit :

$$
\varepsilon_{t}=\varepsilon_{1}+\left(\varepsilon_{n}-\varepsilon_{1}\right) \frac{\lg 100 \text { ans }-\lg t_{1}}{\lg t_{n}-\lg t_{1}} .
$$




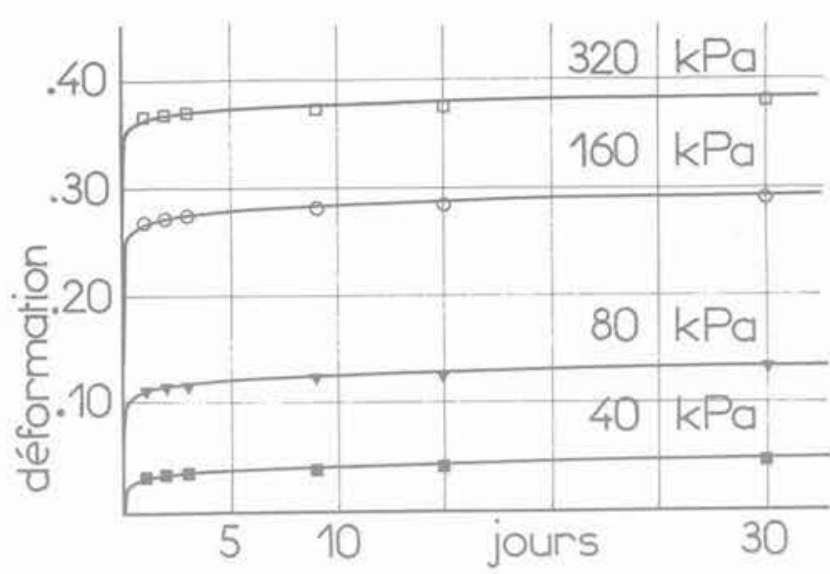

Fig. 4 Mesures de fluage correspondant à différentes charges constantes

\section{$\log$ i (minutes)}

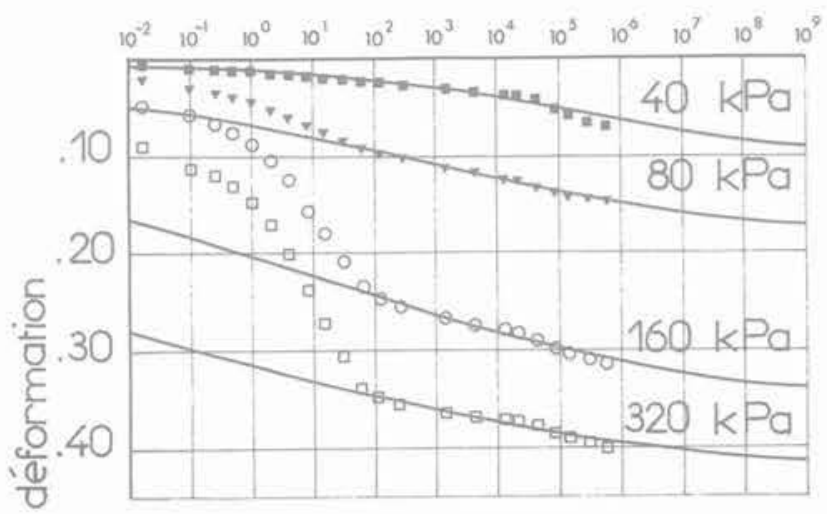

Fig. 5 Mesures de fluage représentées en fonction du logarithme du temps

Fig. 6 La fonction de contrainte $f\left(\sigma^{\prime}\right)$

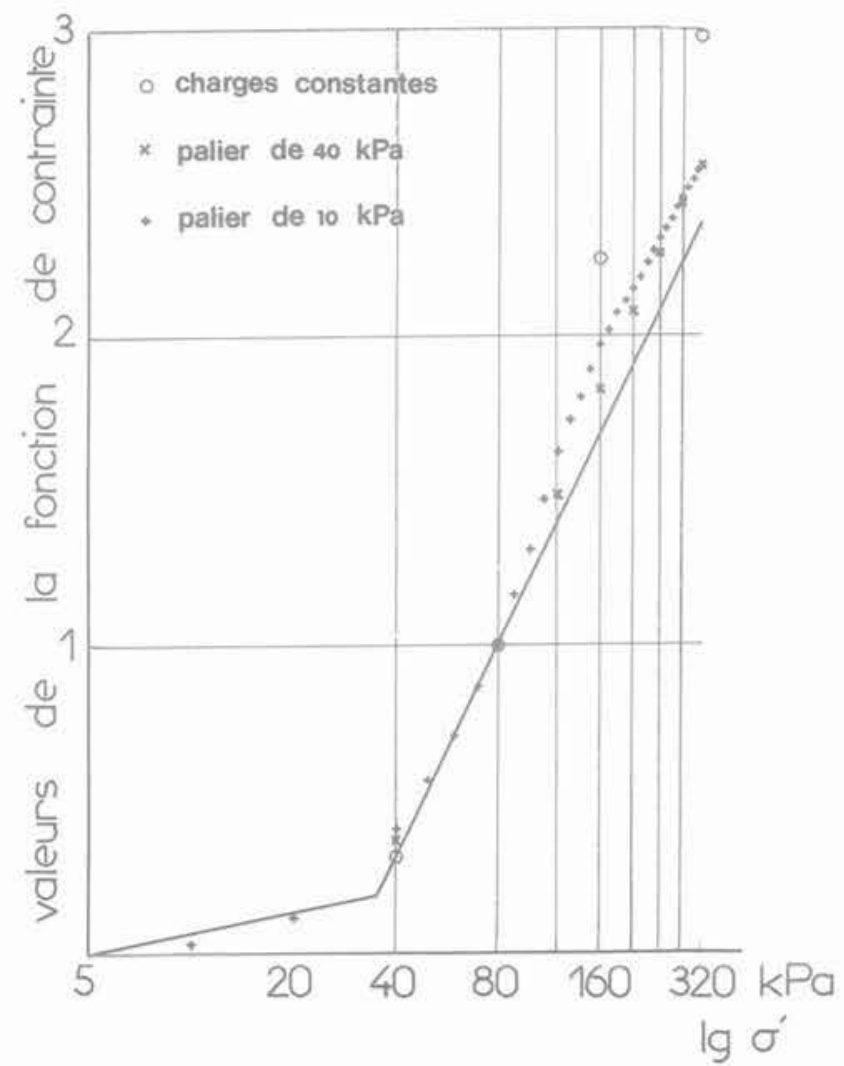

Les déformations au temps conventionnel $t_{0}$, provoquées par une charge constante queiconque $\sigma_{i}^{\prime}$ et par une charge unité, suffisent pour déterminer les quatre paramètres de la fonction de contrainte. Aux trois conditions (32) s'ajoute la relation (26) écrite pour la contrainte $\sigma_{i}^{\prime}$.

La figure 6 présente la fonction de contrainte déterminée de cette façon.

II existe également une méthode approchée de détermination de ces paramètres qui utilise les résultats d'un essai de fluage sous une série de $n$ paliers d'une durée $\Delta t_{i}=t_{i}-t_{i-1}$ quelconque, mais toujours supérieur à un certain intervalle de temps $\Delta T$ donné. La charge est portée instantanément au temps $\mathrm{t}_{\mathrm{i}}$ de la valeur $\sigma_{i}^{\prime}$ à $\sigma_{i+1}^{\prime}$.

Le régime de chargement est décrit par la fonction :

$$
\sigma^{\prime}(t)=\sigma_{1}^{\prime} h(t)+\sum_{i=1}^{n-1}\left(\sigma_{i+1}^{\prime}-\sigma_{i}^{\prime}\right) h\left(t-t_{i}\right)
$$

où $h(t)$ est la fonction échelon de Heaviside :

$$
h(t)=0 \text { pour } t<0 \text { et } h(t)=1 \text { pour } t>0 \text {. }
$$

La fonction de contrainte correspondante s'écrit :

$$
f\left[\sigma^{\prime}(t)\right]=f\left(\sigma_{1}^{\prime}\right) h(t)+\sum_{i=1}^{n-1}\left[f\left(\sigma_{i+1}^{\prime}\right)-f\left(\sigma_{i}^{\prime}\right)\right] h\left(t-t_{i}\right)
$$

et la déformation :

$$
\begin{aligned}
& \varepsilon(t)=\int_{0}^{t} K(t-\tau) f\left(\sigma_{1}^{\prime}\right) h(\tau) d \tau \\
& +\sum_{i=1}^{n-1} \int_{0}^{t} K(t-\tau)\left[f\left(\sigma_{i+1}^{\prime}\right)-f\left(\sigma_{i}^{\prime}\right)\right] h\left(\tau-t_{i}\right) d \tau \\
& \varepsilon(t)=f\left(\sigma_{1}^{\prime}\right) C(t)+\sum_{i=1}^{n-1}\left[f\left(\sigma_{i+1}^{\prime}\right)-f\left(\sigma_{i}^{\prime}\right)\right] C\left(t-t_{i}\right) .
\end{aligned}
$$

Si l'intervalle de temps $\Delta T$ est suffisamment grand (5 à 6 jours minimum), la mesure de fluage peut être considérée comme stabilisée c'est-à-dire :

pour $\quad t \geqslant \Delta T ; \quad C(t)=C(\Delta T)$,

et l'équation (35) se réduit à :

$$
\varepsilon\left(t_{n}\right)=f\left[\sigma_{n}^{\prime}\right] C(\Delta T) \text {. }
$$

De la déformation mesurée à la fin de chaque palier, on peut déduire la valeur de la fonction de contrainte pour la contrainte $\sigma_{n}$ correspondante.

Meschyan (1967) a proposé une méthode analogue mais moins générale. Sur la figure 6 sont représentés les points calculés par la formule (36), à partir des résultats de deux essais de fluage sous des paliers de charge égaux au huitième et à la moitié de la charge unité, appliqués à intervalles de 14 jours.

Si une approximation plus grossière est admise, les paramètres de la fonction de contrainte peuvent être déduits des résultats d'un essai classique de mesure de la compressibilité ( $\Delta T=24$ heures). L'application d'une charge unité $\sigma_{1}^{\prime}$ sur un sol à l'équilibre sous la charge initiale $\sigma_{0}^{\prime}$, provoque une variation $\Delta \mathrm{e}_{1}$ de l'indice des vides (fig. 7).

Les paramètres de la fonction de contrainte sont liés à ceux de la courbe de compressibilité par les relations :

$$
\begin{gathered}
a_{1}=\frac{C_{s}}{\Delta e_{1}} ; \quad b_{1}=\frac{C_{s} \lg \sigma_{0}^{\prime}}{\Delta e_{1}} ; \quad a_{2}=\frac{C_{c}}{\Delta e_{1}} ; \\
b_{2}=\frac{\left(C_{c}-C_{s}\right) \lg \sigma_{p}^{\prime}+C_{s} \lg \sigma_{0}^{\prime}}{\Delta e_{1}} .
\end{gathered}
$$




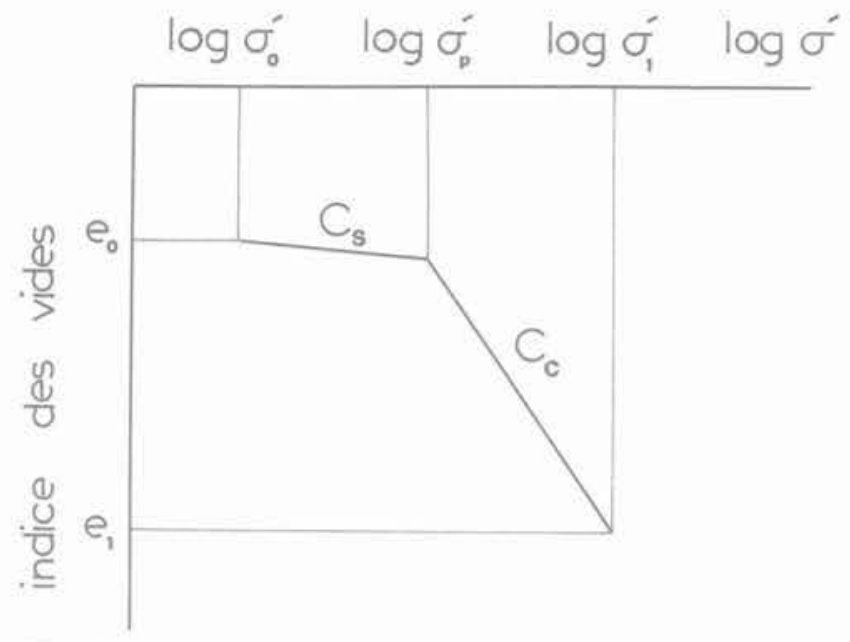

Fig. 7 Variation de l'indice des vides sous la charge unité

\section{Vérification expérimentale}

Les essais ont été réalisés sur l'argile de Cubzac-lesPonts, de couleur gris vert et contenant quelques débris végétaux très décomposés. Les caractéristiques sont récapitulées dans le tableau 1.

\begin{tabular}{l|c|c}
\hline teneur en eau \% & & 83 \\
\hline indice des vides & & 2,3 \\
\hline limite de liquidité & $\mathrm{W}_{\mathrm{L}}$ & 110 \\
\hline limite de plasticité & $\mathrm{W}_{\mathrm{p}}$ & 50 \\
\hline indice de compression & $\mathrm{C}_{\mathrm{c}}$ & 0,98 \\
\hline indice de gonflement & $\mathrm{C}_{\mathrm{s}}$ & 0,11 \\
\hline contrainte de préconsolidation & $\sigma_{\mathrm{p}}^{\prime}$ & $35 \mathrm{kPa}$ \\
\hline
\end{tabular}

\section{Tableau 1 Caractéristiques des échantillons utilisés}

\subsection{Influence de la longueur de drainage}

D'après la théorie du fluage héréditaire, l'application ou le retrait momentané d'une charge exerce une influence sur la déformation, qui décroît dans le temps avec la fonction $\mathrm{K}(\mathrm{t})$, et devient rapidement nulle. Dans le cas du squelette intergranulaire des sols argileux, et si les hypothèses précédentes sont vérifiées, le retard apporté au chargement par les surpressions interstitielles n'a pas d'influence sur les déformations de consolidation secondaire. II est relativement facile de vérifier si la distance de drainage, qui détermine la durée de la phase hydrodynamique, a une influence sur la phase secondaire.

Quatre échantillons dont les distances de drainage $\mathrm{h}$ s'échelonnent suivant les valeurs $5,10,20$ et $40 \mathrm{~mm}$, ont été reconsolidés sous $\sigma_{\mathrm{p}}^{\prime}$, puis soumis à quatre paliers de charges de $40 \mathrm{kPa}$ d'une durée de 8 jours.

Les figures 8 et 9 représentent, en fonction du logarithme du temps, les déformations provoquées par le premier et le dernier palier en considérant comme initiale la hauteur des échantillons avant l'application de chaque palier.

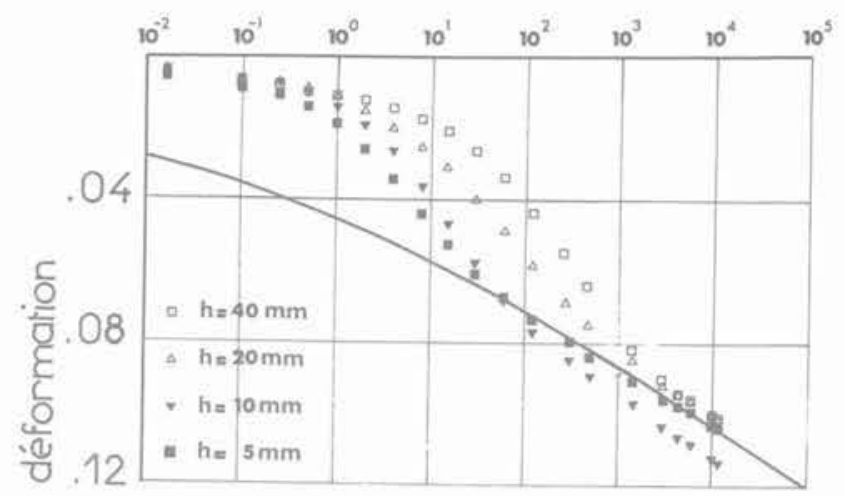

Fig. 8 Consolidation et fluage de quatre échantillons ayant des longueurs de drainage différentes (fortes charges)

\section{$\log t$ (minutes)}

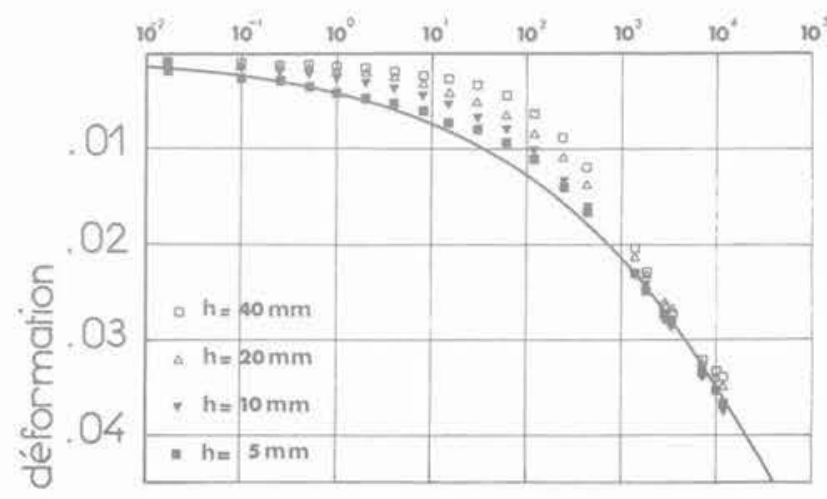

Fig. 9 Consolidation et fluage de quatre échantillons ayant des longueurs de drainage différentes (faibles charges)

Dans les deux cas, les déformations mesurées après 48 heures présentent une dispersion très limitée, sur laquelle la longueur de drainage n'a pas d'influence apparente.

Ces essais montrent, d'une part, que les déformations de consolidation secondaire ne sont pas dues à un écoulement différé du liquide interstitiel, mais aux caractéristiques visqueuses du squelette. Les paramètres des fonctions $C(t)$ et $f\left(\sigma^{\prime}\right)$ qui les caractérisent déterminent une loi de comportement qui peut, a priori, être extrapolée à la phase hydrodynamique de la consolidation.

D'autre part, les essais montrent que I'hypothèse B présentée par Ladd (1977), est conforme à l'évolution réelle de la consolidation de monocouches d'épaisseurs différentes; et non pas I'hypothèse $A$, d'après laquelle la déformation en fin de consolidation primaire est toujours la même quelle que soit la longueur de drainage.

\subsection{Affinité des courbes de fluage sous charges constantes}

La formule (25), issue de la théorie du fluage héréditaire non linéaire, montre que les courbes de fluage sous charge constante se déduisent de la courbe mesure de fluage représentative de $C(t)$, par affinité orthogonale de coefficient $f\left(\sigma^{\prime}\right)$. 


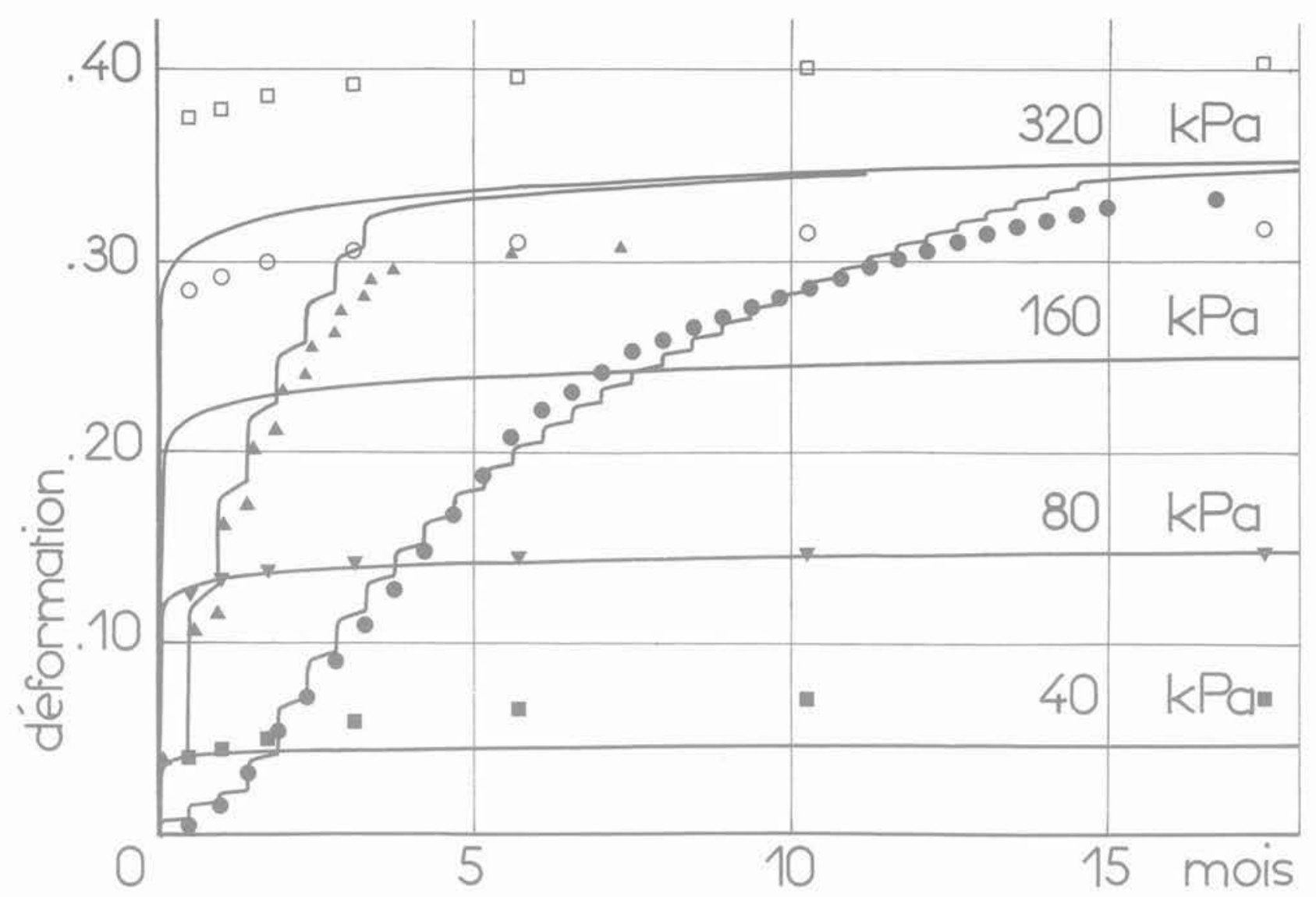

résultats expérimentaux

charges constantes $\square 320 \mathrm{kPa} \quad \circ 160 \mathrm{kPa} \quad \nabla 8 \mathrm{kPa}=40 \mathrm{kPa}$
chargement par paliers égaux à

Fig. 10 Fluage œdométrique sous différentes charges appliquées instantanément ou par paliers

Aux quatre charges constantes $40-80-160$ et $320 \mathrm{kPa}$ correspondent des courbes dont les paramètres $\alpha$ et $\beta$, calculés par la méthode exposée au chapitre 2.2 , sont indiqués sur le tableau 2.

\begin{tabular}{c|c|c|c}
\hline $\begin{array}{c}\text { charge } \\
\text { appliquée }\end{array}$ & $\begin{array}{c}\text { déformation } \\
\text { finale } \%\end{array}$ & $\begin{array}{c}\alpha \text { (pour } \\
\text { ten min.) }\end{array}$ & $\beta$ \\
\hline $40 \mathrm{kPa}$ & 9,3 & 0,1086 & 0,182 \\
\hline $80 \mathrm{kPa}$ & 17,1 & 0,5033 & 0,103 \\
\hline $160 \mathrm{kPa}$ & 34,8 & 0,8488 & 0,0745 \\
\hline $320 \mathrm{kPa}$ & 42,7 & 1,267 & 0,0567 \\
\hline
\end{tabular}

Tableau 2 Paramètres des fonctions mesure de fluage associées à quatre charges constantes
Aux grandes charges correspondent les plus grandes valeurs de $\alpha$, c'est-à-dire les croissances les plus rapides vers la valeur finale $\varepsilon_{t}$, et les plus petites valeurs de $\beta$ c'est-à-dire les vitesses les plus grandes dans la période initiale. En fait, plus la charge appliquée est grande, plus la composante quasiinstantanée, qui se développe surtout au cours de la consolidation hydrodynamique, est importante par rapport à la composante différée (Léonards et Girault, 1961).

La théorie du fluage héréditaire ne peut pas tenir compte de cette variation des paramètres $\alpha$ et $\beta$. Ceux retenus pour l'établissement de l'équation de comportement sont relatifs à la charge choisie comme unité $\sigma_{1}^{\prime}=80 \mathrm{kPa}$. Sur les figures 10 et 11 sont tracées, conformément à cette équation, les courbes relatives aux autres charges.

Pour les charges 40 et $80 \mathrm{kPa}$ impliquées dans la détermination des fonctions $\mathrm{C}(\mathrm{t})$ et $\mathrm{f}\left(\mathrm{\sigma}^{\prime}\right)$, l'approximation est correcte dans les 60 premiers jours. Apparaît ensuite une instabilité de la vitesse de fluage qui a été remarquée au cours de tous les essais œdométriques de longue durée (Bishop, Lovenbury 1969; Tavenas et al, 1978). 


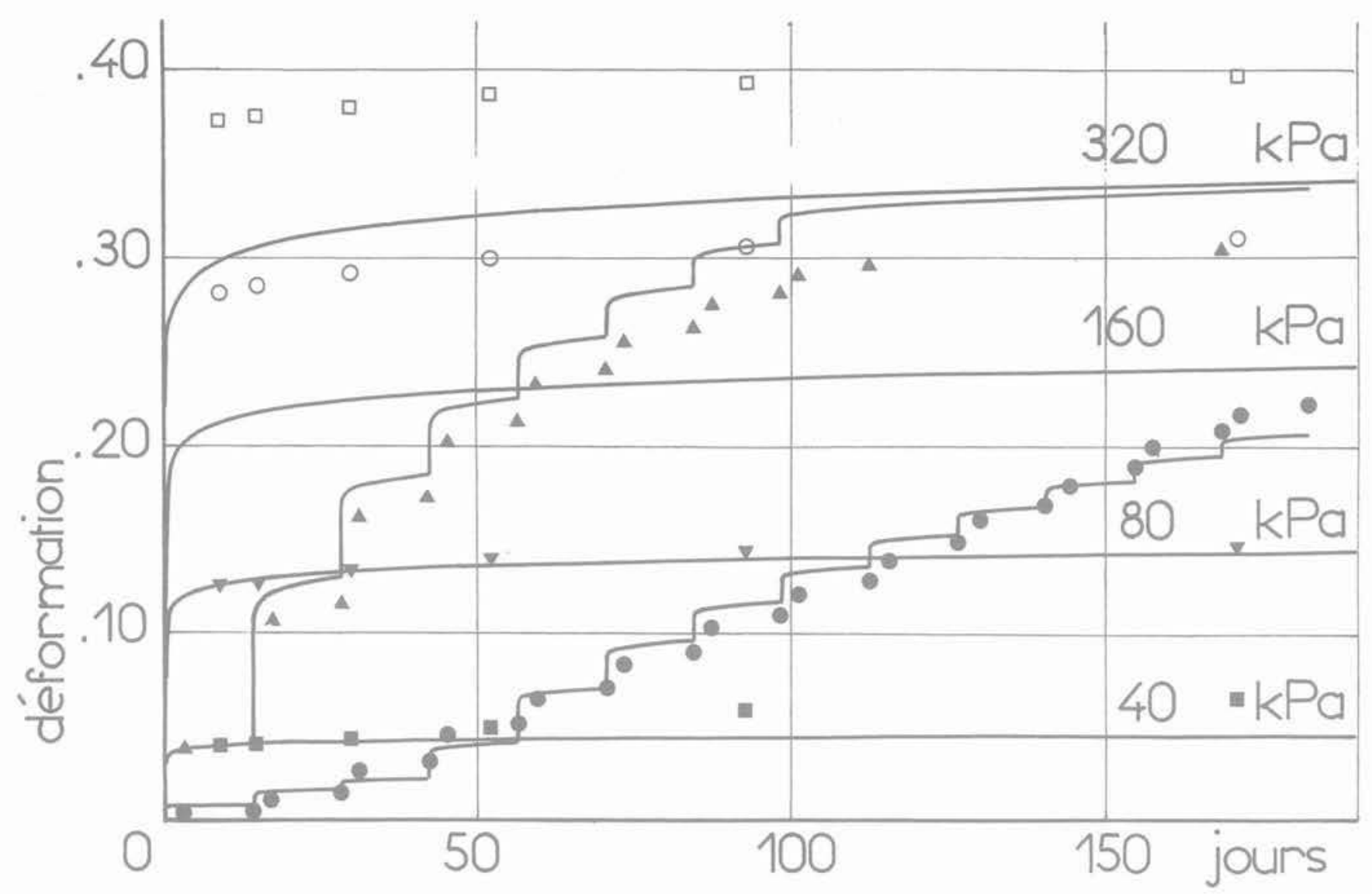

résultats expérimentaux

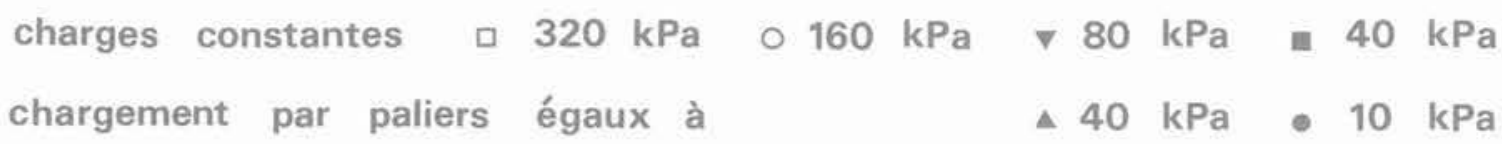

Fig. 11 Fluage codométrique sous différentes charges appliquées instantanément ou par paliers (période initiale)

Par contre, un écart relatif de l'ordre de $25 \%$ existe entre les déformations calculées et mesurées pour les charges de 160 et $320 \mathrm{kPa}$. II reste approximativement constant dans le temps, par conséquent l'hypothèse de l'affinité n'est pas mise en défaut, et ne peut expliquer cet écart. Les essais indiquent que les chargements les plus importants (supérieurs à $120 \mathrm{kPa}$ ), appliqués instantanément, provoquent un surcroît de déformation, essentiellement plastique. II convient de les écarter du domaine de validité de l'équation de comportement.

\subsection{Principe de superposition}

Deux essais de fluage sous des paliers de charge égaux, appliqués à intervalles réguliers de 14 jours, ont été réalisés. La première série comportait 8 paliers de $40 \mathrm{kPa}$ et la deuxième 32 paliers de $10 \mathrm{kPa}$. Si les hypothèses de la théorie du fluage héréditaire, et en particulier le principe de superposition sont vérifiés, l'équation (35) décrit la déformation provoquée. La figure 10 représente l'ensemble des paliers de chargement et la figure 11 , les 8 paliers du premier essai et les
12 premiers du deuxième, avec une échelle des temps beaucoup plus grande. Les points correspondent aux mesures, et les courbes aux calculs. Celles-ci ont été prolongées après la fin du chargement pour représenter la déformation sous la charge finale $320 \mathrm{kPa}$ maintenue. On constate qu'elle converge rapidement vers la déformation qui correspond à la même charge appliquée instantanément. En effet, d'après la théorie du fluage héréditaire, l'influence du mode d'application de la charge s'estompe dans le temps.

L'écart relatif entre les déformations calculées et mesurées est de l'ordre de $10 \%$. II est suffisamment petit pour que le domaine de validité de l'équation de comportement puisse être étendu aux chargements croissant par paliers. Dans la pratique, les contraintes effectives appliquées au squelette intergranulaire des sols argileux, suivent très souvent de telles croissances lentes et monotones, ne serait-ce que pendant la phase hydrodynamique de la consolidation.

Par contre, la validité de l'équation de comportement ne s'étend pas aux cas de chargements comprenant des phases alternées de croissance et de décroissance. Un exemple est fourni par un essai de compressibilité conforme au projet de nouveau mode opératoire des 


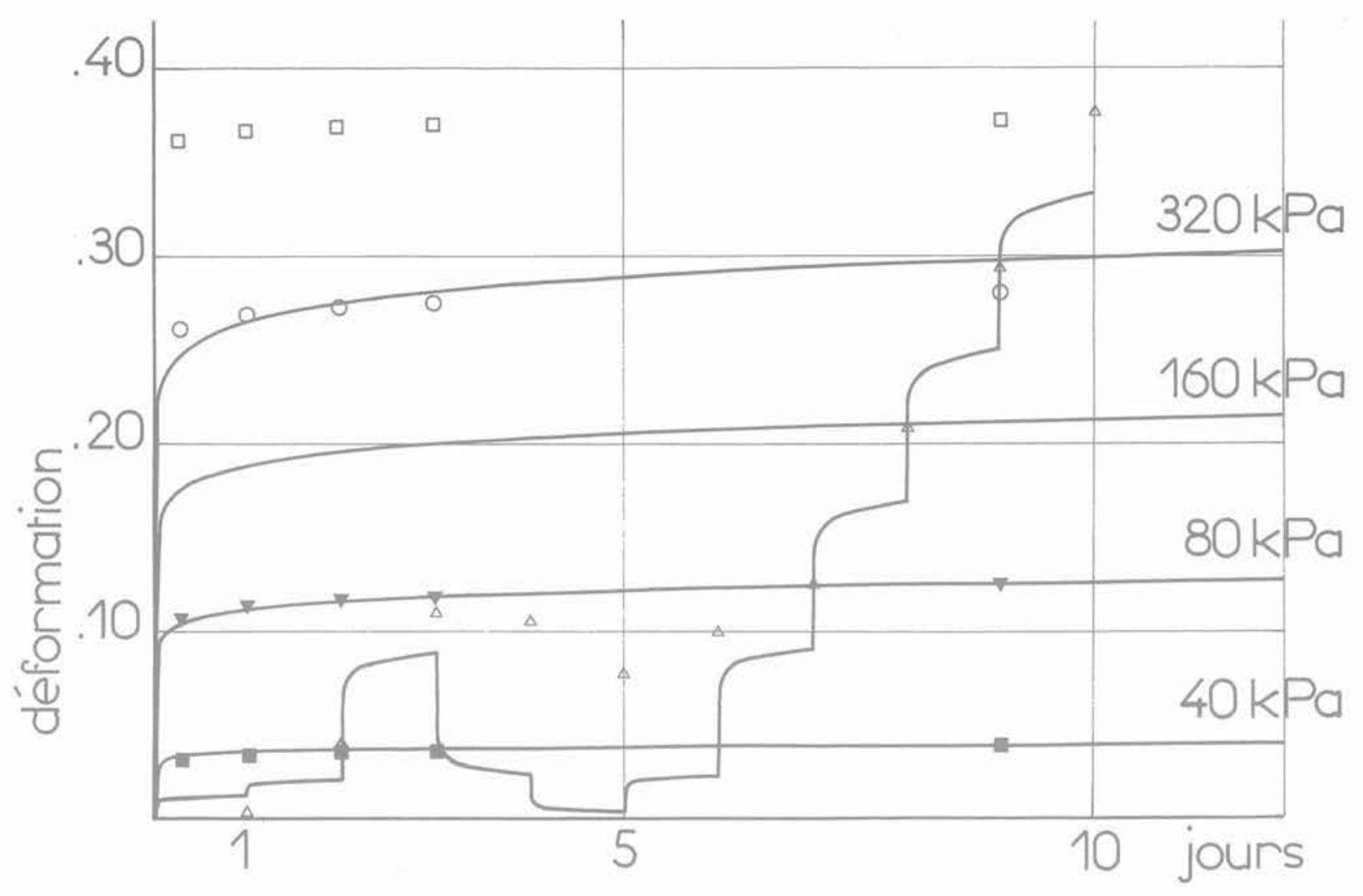

résultats expérimentaux

charges constantes ㅁ $320 \mathrm{kPa} \circ 160 \mathrm{kPa} \nabla 80 \mathrm{kPa}=40 \mathrm{kPa}$
essai conforme au projet de nouveau mode opératoire des LPC $\triangle$

Fig. 12 Fluage sous un cycle de chargement suivi d'un rechargement

LPC. Les déformations mesurées et calculées en fonction du temps par l'équation (35) sont représentées comme précédemment sur la figure 12. La théorie du fluage héréditaire suppose que la déformation est entièrement réversible, et s'annule après déchargement complet. La déformation réelle ne suit pas la même évolution car même petite, elle est en partie plastique (irréversible).

\section{Conclusion}

Le modèle issu de la théorie du fluage héréditaire non linéaire est caractérisé par la forme mathématique de deux fonctions. La fonction de contrainte est analogue à la relation logarithmique classique qui lie contrainte et déformation. La mesure de fluage, plus originale, est reprise des travaux de Kohlrausch. Les paramètres du modèle sont calculés à partir des déformations de consolidation secondaire mesurées sous charges constantes. Les essais ont montré qu'elles ne dépendaient pas de la durée de la consolidation primaire. Le fluage est dû aux caractéristiques visqueuses du squelette et l'extrapolation du modèle au comportement de celui-ci durant la phase primaire de la consolidation est a priori licite. Le domaine de validité s'étend à des charges de grande amplitude, pourvu que celles-ci soient appliquées lentement et suivant une croissance monotone, comme cela se produit au cours de la consolidation hydrodynamique des sols de fondation. L'utilisation du modèle, en tant qu'équation d'état du squelette intergranulaire dans une théorie de la consolidation unidimensionnelle, peut donc être envisagée. 


\section{Références bibliographiques}

ARUTYUNYAN N. Kh. (1952) Quelques questions sur la théorie du fluage (en russe), édition Gostekhizdat Moscou Leningrad.

BARDEN L., (1965) Consolidation of clay with nonlinear viscosity, Géotechnique, Vol. XV, $n^{\circ} 4$, décembre 1965, pp. 345-362.

BARDEN L., (1969) Time-dependant deformation of normally consolidated clays and peats, journal of the Soil Mechanics and Foundations division-Proceedings of the A. S. C. E., Vol. 95, n SM 1, janvier 1969, p. 1-31.

BATTELINO D., (1973) Oedometer testing of viscous soils, août 1973, Proceedings of the 8 th International Conference on Soil Mechanics and Foundation Engineering, Moscou. Vol. 1-1, p. 25-30.

BISHOP A. W.: LOVENBURY H.T., (1969) Creep characteristics of two undisturbed clays, août 1969, Proceedings of the 7 th International Conference on Soil Mechanics and Foundation Engineering, Mexico. Vol. 1, p. 29-38.

BJERRUM L., (1967) 7 th Rankine lecture Engineering geology of normally consolidated marine clays as related to settlements of buildings, Géotechnique, Vol. XVII, $\mathrm{n}^{\circ} 2$, juin 1967, p. $82-118$.

BUISMAN A. S. K., (1936) Results of long duration settlements tests, juin 1936, Proceedings of the 1 st International Conference on soil Mechanics and Foundation Engineering, Cambridge Mass. U.S.A., Vol. 1, nF7, p. 103-106.

FELIX B., (1980) Le fluage des sols argileux, étude bibliographique, Rapport de Recherche L.P.C., $n^{\circ} 93$, à paraître, Paris, 234 pages.

FELIX B., (1980) Fluage et consolidation unidimension nelle des sols argileux, Rapport de Recherche L.P.C., $n^{\circ} 94$, à paraître, Paris, 180 pages.

FLORIN V. A., (1953) La consolidation unidimensionnelle d'un sol avec prise en compte du vieillissement. du fluage non linéaire, et de la destruction de la structure (en russe), édition de l'Académie des Sciences de I'U.R.S.S., O.T.N. $n^{\circ} 9$.

FLORIN V.A., (1961) Les bases de la mécanique des sols tome II (en russe), édition Gosstrojizdat, 1961, Moscou, 543 pages.

GARLANGER J.E., (1972) The consolidation of soils exhibiting creep under constant effective stress, Géotechnique, Vol. XXII, $\mathrm{n}^{\circ} 1$, mars 1972 , p. 71-78.

GIBSON R. E.; LO K. Y., (1961) A theory of consolidation for soils exhibiting secondary compression, publication $n^{\circ} 41$, Norwegian Geotechnical Institut, p. 3-15.

HANSEN B., (1969) A mathematical model for creep phenomena in clay, août 1969, Proceedings of the 7 th International Conference on Soil Mechanics and Foundation Engineering, Mexico, Speciality Session $n^{\circ} 12$ Advances in consolidation theories for clays, p. $12-18$.
HAWLEY J.G., BORIN D. L., (1973) A unified theory for the consolidation of clays, aout 1973, Proceedings of the 8 th International Conference on Soil Mechanics and Foundation Engineering, Moscou, Vol. 1-3 p. 107120.

KOHLRAUSCH F., (1863) Experimentale Untersuchun gen über die elastische Nachwirkung bei der Torsion, Ausdehnung und Biegung, Pogg. Ann. Bd 119.

KOPPEJAN A.W., (1948) A Formula combining the Terzaghi's load compression relationship and the Buisman's secular time effect, juin 1948, Proceedings of the 2 nd International Conference on Soil Mechanics and Foundation Engineering, Rotterdam, Vol. 3, p. 32 37.

LADD C.C. et al, (1977) Stress-deformation and strength characteristics (State-of-the-art Report), juillet 1977, Proceedings of the 9 th International conference on Soil Mechanics and Foundation Engineering, Tokyo, Vol. 2, p. 421-494.

LEONARDS G. A.; GIRAULT P., (1961) A study of the one-dimensional consolidation test, juillet 1961, Proceedings of the 5 th International Conference on Soil Mechanics and Foundation Engineering, Paris, Vol. 1, p. 213-218.

MAGNAN J.-P. et al, (1979) Étude numérique de la consolidation unidimensionnelle en tenant compte des variations de la perméabilité et de la compressibilité du sol, du fluage et de la non saturation, bull. de liaison des Labo des Ponts et Chaussées, $n^{\circ} 103$, septembreoctobre 1979 , p. $83-94$

MASLOV G. N., (1940) Etat de contrainte et de température dans les massifs en béton avec prise en compte du fluage du béton (en russe), bulletin du VNIIG, Vol. 28.

MESCHYAN S.R., (1967) Le fluage des sols argileux (en russe), édition de l'Académie des Sciences de la R.S.S. d'Arménie, 1967, 318 pages.

MESCHYAN S. R., (1969) Sur les lois décrivant les processus de déformation des sols argileux dans le temps, journal Soil Mechanics and Foundation Engineering traduction anglaise du journal Osnovaniya Fundamenty i Mekhanika Gruntov, janvier-février 1969, $n^{\circ} 1$, p. $10-15$

MESCHYAN S.R., (1974) Les propriétés mécaniques des sols et leur détermination au laboratoire (avec prise en compte des effets du temps) en russe, édition Nedra-Moscou, 190 pages.

MESRI G.; ROKHSAR A., (1974) Theory of consolidation of clays, Journal of the Geotechnical Engineering Division, Proceedings of the A.S.C.E., Vol. 100, GT8, août 1974, p. 889-904.

MESRI G., GODLEWSKI P.M., (1977) Time and stress compressibility interrelationship, Journal of the Geotechnical Engineering division, Proceedings of the A.S.C.E., Vol. 103, GT5, mai 1977, p. 417-430.

MIEUSSENS C., (1979) Étude en laboratoire de la compressibilité des sols fins - Essais de fluage à très long terme - Étude de l'effet d'échelle dans l'essai œométrique. Rapport de recherche, L. R. de Toulouse des Ponts et Chaussées, mars 1979. 
MIEUSSENS C.; MAGNAN J.-P.; VAUTRAIN J., Le nouveau mode opératoire des Laboratoires des Ponts et Chaussées pour l'essai de compressibilité à l'œdomètre, bull. de liaison des laboratoires des Ponts et Chaussées à paraitre.

MURAYAMA S.; SHIBATA T., (1964) Flow and stress relaxation of clays, avril 1964. Symposium de I'I.U.T.A.M. Rhéologie et mécanique des sols, Grenoble, p. 99-129.

POKROVSKIJ G. I., (1933) Application du principe de Boltzmann au calcul du tassement des fondations (en russe), dans le recueil * osnovaniya i fundamenty $\approx n^{\circ} 1$ V.I.O.S. Moscou-Leningrad.

POOROOSHASB H. B.; SIVAPATHAM T., (1969) Consolidation of sensitive clays exhibiting strong structural breakdown aout 1969, Proceedings of the 7 th International conference on Soil Mechanics and Foundation Engineering, Mexico, Speciality Session $n^{\circ} 12$, Advances in consolidation theories for clays, p. 27-37.

POSKITT J.; BIRDSALL J.O., (1971) A theoretical and experimental investigation of mildly non-linear consolidation behaviour in saturated soil, Canadian geotechnical journal, Vol. $8, n^{\circ} 2$, mai 1971, p. 186-216.

POSKITT T. J., (1971) Consolidation of clay and peat with variable properties. Journal of the Soil Mechanics and Foundations Division-Proceedings of the A.S.C.E. Vol. 9, $n^{\circ}$ SM6, Juin 1971, p. 841-880.

RABOTNOV Yu N., (1948) Quelques questions sur la théorie du fluage (en russe), nouvelles de l'université d'état de Moscou (MGU) $n^{\circ} 10$.

SCHIFFMAN L., (1969) Secondary consolidation of clay, a visco elastic analysis août 1969. Proceedings of the 7 th International Conference on Soil Mechanics and Foundation Engineering, Mexico, Speciality Session $n^{\circ} 12$, Advances in consolidation theories for clays, p. $60-73$.

SUUKLJE L., (1957) The Analysis of the Consolidation Process by the Isotache Method, aout 1957, Proceedings of the 4 th International Conference on Soil Mechanics and Foundation Engineering, Londres, Vol. I, pp. 200-206, discussions Vol. III, pp. 107-109.

ŠUKLJE L., (1969) Rheological aspects of Soil Mechanics, édition Wiley interscience, Londres, 1969 , 571 pages.

ŠUKLJE L.; KOVAČIČ I., (1974) The role of effective stress speed in the consolidation analysis. Acta geotechnica, 51-53, 1974, Université de Ljubljana, p. $233-240$.
ŠUKLJE L.; KOZAK J., (1974) Consolidation of partly saturated viscous soils, Acta geotechnica, 54-56, 1974 . Université de Ljubljana, p. 1-20.

TAN TJONG KIE, (1958) Secondary Time effects and Consolidation of clays, Scientia Sinica, Vol. 7, $\mathrm{n}^{\circ} 11$, p. 1060 .

TAVENAS F. et al, (1978) Creep behaviour of an undisturbed lightly overconsolidated clay, Canadian geotechnical journal, Vol. $15, n^{\circ} 3$, août 1978 , p. 402 423.

TAYLOR D. W.; MERCHANT W.A., (1940) A theory of clay consolidation accounting for secondary compres sion, journal of mathematics and physics, Vol. $19, n^{\circ} 3$, p. 167-185.

TAYLOR D.W. (1942) Research on Consolidation of clays Massachusetts Institute of Technology, Cambridge U.S. A., publication from the Department of civil and Sanitary Engineering, Serial 82 août 42.

TER MARTIROSYAN Z.G.; TSYTOVICH N.A., (1965) Sur la consolidation secondaire des argiles, journal Soil Mechanics and Foundation Engineering, 1965, $n^{\circ} 5$, traduction anglaise du journal osnovaniya fundamenty i mekhanika gruntov, septembreoctobre $1965, n^{\circ} 5$, p. 12-15.

TSYTOVICH N.A. et al, (1967) Prévision de la vitesse des tassements des fondations des ouvrages (en russe). Edition literatury po stroitel'stvu, Moscou 1967, 239 pages.

VU CAO MINH, (1977) One-dimensional consolidation of soils taking creep into account, Archiwum hydrotechniki Varsovie, tome XXIV, 1977, p. 187-203.

WU T.H.; RESENDIZ D.; NEUKIRCHNER R.J., (1966) The analysis of consolidation by rate process theory, Journal of the Soil Mechanics and Foundations Division, Proceedings of the A.S.C. E., Vol. 92, $n^{\circ}$ SM6, novembre 1966 , p. 229-248.

ZARETSKIJ Yu. K., (1967) Théorie de la consolidation des sols (en russe), édition Nauka, Moscou 1967. 270 pages, traduction en anglais Israël programm of scientific translation, Jérusalem 1972 (réf. DIRR 22124). 